\section{Metabolische Basisdiagnostik}

\section{G. F. Hoffmann ${ }^{1}$, C.-D. Langhans ${ }^{2}$ und A. Schulze ${ }^{3}$}

${ }^{1}$ Zentrum für Kinder- und Jugendmedizin, Universitätsklinikum Heidelberg, Heidelberg, Deutschland ${ }^{2}$ Stoffwechsellabor - GCMS, Universitätsklinik für Kinderund Jugendmedizin, Heidelberg, Deutschland ${ }^{3}$ Clinical and Metabolic Genetics The Hospital for Sick Children, University of Toronto, Toronto, Kanada

Beschreibung Bei klinischem Verdacht auf das Vorliegen einer entgleisten Stoffwechselerkrankung geben einige Laborparameter des klinisch-chemischen Routinelabors bereits erste differenzialdiagnostische Hinweise für eine gezielte metabolische Diagnostik und Initialtherapie. Die Tabelle gibt einen Überblick über die metabolische Basisdiagnostik:

\begin{tabular}{|c|c|c|}
\hline Laborparameter & Konzentration & Stoffwechselerkrankung \\
\hline Ammoniak & Erhöht & $\begin{array}{l}\text { Harnstoffzyklusdefekte } \\
\text { Organoazidopathien } \\
\text { Fettsäurenoxidationsdefekte } \\
\text { Mitochondriopathien }\end{array}$ \\
\hline \multirow[t]{2}{*}{ Blutgase } & Alkalose & Harnstoffzyklusdefekte \\
\hline & Acidose & $\begin{array}{l}\text { Organoazidopathien } \\
\text { Fettsäurenoxidationsdefekte } \\
\text { Mitochondriopathien }\end{array}$ \\
\hline Anionenlücke & Erhöht & $\begin{array}{l}\text { Organoazidopathie } \\
\text { Fettsäurenoxidationsdefekte } \\
\text { Mitochondriopathien }\end{array}$ \\
\hline \multirow[t]{2}{*}{ Blutzucker } & Erniedrigt & $\begin{array}{l}\text { Endokrinopathien } \\
\text { Glykogenosen } \\
\text { Glukoneogenesedefekte } \\
\text { Fettsäureoxidationsstörungen } \\
\text { (Organoazidopathien, } \\
\text { Mitochondriopathien) }\end{array}$ \\
\hline & Erhöht & $\begin{array}{l}\text { Endokrinopathien } \\
\text { (Mitochondriopathien, } \\
\text { Organoazidopathien) }\end{array}$ \\
\hline
\end{tabular}

(Fortsetzung)

\begin{tabular}{|c|c|c|}
\hline Laborparameter & Konzentration & Stoffwechselerkrankung \\
\hline Laktat & Erhöht & $\begin{array}{l}\text { Mitochondriopathien } \\
\text { Organoazidopathien } \\
\text { Fettsäureoxidationsstörungen } \\
\text { Glykogenosen } \\
\text { Glukoneogenesedefekte }\end{array}$ \\
\hline \multirow[t]{2}{*}{ Harnsäure } & Erhöht & $\begin{array}{l}\text { Purinstoffwechseldefekte } \\
\text { Glykogenosen } \\
\text { Fettsäureoxidationsstörungen } \\
\text { Mitochondriopathien }\end{array}$ \\
\hline & Erniedrigt & $\begin{array}{l}\text { Purinstoffwechseldefekte } \\
\text { (z. B. Molybdän- } \\
\text { Kofaktormangel) }\end{array}$ \\
\hline Kreatinin & Erniedrigt & $\begin{array}{l}\text { Kreatinsynthesedefekt } \\
\text { (z. B. GAMT-Mangel) }\end{array}$ \\
\hline Triglyzeride & Erhöht & $\begin{array}{l}\text { Glykogenosen, Störungen im } \\
\text { Lipoproteinstoffwechsel }\end{array}$ \\
\hline $\mathrm{CK}$ & Erhöht & $\begin{array}{l}\text { Fettsäureoxidationsstörungen } \\
\text { Mitochondriopathien } \\
\text { Glykogenosen } \\
\text { Glykolysedefekte }\end{array}$ \\
\hline GOT, GPT & Erhöht & $\begin{array}{l}\text { Fettsäureoxidationsstörungen } \\
\text { Harnstoffzyklusdefekte } \\
\text { Mitochondriopathien } \\
\text { CDG-Syndrome } \\
\text { Peroxisomale } \\
\text { Erkrankungen }\end{array}$ \\
\hline Cholesterol & Erniedrigt & $\begin{array}{l}\text { Sterolsynthesedefekte } \\
\text { Peroxisomale } \\
\text { Erkrankungen }\end{array}$ \\
\hline
\end{tabular}

\section{Literatur}

Zschocke J, Hoffmann GF (2012) Vademecum Metabolicum - Diagnose und Therapie erblicher Stoffwechselkrankheiten, 4. Aufl. MilupaSchattauer Verlag, Stuttgart 